\title{
Current Challenges and Future Perspectives for Applying Biologically Synthesized Magnetic Nanoparticles for Human Health Benefit
}

\author{
Gabriele Vargas and Fernanda Abreu* \\ Instituto de Microbiologia Paulo de Góes, Brazil
}

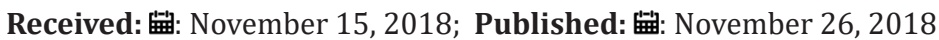

*Corresponding author: Fernanda Abreu, Instituto de Microbiologia Paulo de Góes, Brazil

\begin{abstract}
In modern science, nanoparticles are considered the next-generation tools of biotechnology. Among the diverse qualities of nanoparticles, the interest of the scientific community in magnetic nanoparticles has always been eminent because of the possibility of manipulating these structures by a magnetic field. Magnetic iron oxide nanoparticles are one of the most relevant tools for biotechnology, and these are usually synthesized by chemical methods. A recent and environmentally friendly method to produce biocompatible magnetic nanoparticles is achieved by the growth of magnetotactic bacteria in large scale. Many advantages of using the biologically synthesized nanoparticles have already been showed, including their higher efficiency on drug delivery and on killing tumors by hyperthermia. Therefore, new proposals for the treatment of human diseases using biologically synthesized nanoparticles are growing in the last few years. In the meantime, discussion about the necessary steps to enable the use of these nanoparticles in health treatments is relatively near to the ground. Here we emphasized future challenges to the use of magnetic nanoparticles in biomedicine and proposed alternative tests to complement research dedicated to the evaluation of new biomedical applications on this subject.
\end{abstract}

Keywords: Magnetic Nanoparticles; Magnetosome; Fe304; Iron Oxides; Biomedicine; Biotechnology; Cytotoxicity

Abbreviations: MTT: 3-(4,5-dimethylthiazol-2-yl)-2,5-diphenyltetrazolium bromide; LDH: Lactate dehydrogenase

\section{Introduction}

Nanoparticles technology is usually associated with modern science to ensure human benefits. However, their relevance in the production of glass, ceramic and weaponry dates from ancient history. The famous Lycurgus cup, for example, which was produced by Roman artisans in the $4^{\text {th }}$ century and is now part of the collection of the British Museum in London, shine red or green depending on the direction of the incident light because of the presence of gold and silver nanoparticles dispersed in the glass [1]. The similar dichroic effect can be observed in several stained glass windows in ancient constructions. Another advanced technology of the medieval period was the forge of the Damascus blades, which resulted in swords containing carbon nanotubes and cementite nanowires with outstanding resistance and sharp cutting edge [2]. At that time, artists and forgers did not have techniques to reveal the nanoscale structure of their creations and could hardly imagine that their successes relied on the leading biotechnology tool of the 21 st century, the nanoparticles. From the biomedical point of view, nanoparticles are innovative as next-generation formulations in treatment protocols. The nanoscale particles $(1-100 \mathrm{~nm})$ fall within the same size scale as biomolecules, viruses, cells, and cellular components, allowing their interaction with molecules and cells individually $[3,4]$.

A variety of organic and inorganic nanoparticles with different shapes and chemical nature has been documented [3]. Among them, magnetic nanoparticles are considered ideal tools in approaches ranging from the recovery and guided transport of molecules and metals to a specific location and to the heat generation by the application of an external applied magnetic field [3]. Iron oxides, such as magnetite $\left(\mathrm{Fe}_{3} \mathrm{O}_{4}\right)$ or maghemite $\left(\gamma-\mathrm{Fe}_{2} \mathrm{O}_{3}\right)$ [3], are generally used as nanotools in biomedicine. However, to assure biocompatibility with the human biological system, their surface usually has to be coated with specific modifiers [4]. Nowadays, synthetic superparamagnetic iron oxide nanoparticles of approximately $20 \mathrm{~nm}$ are used as image contrast agents in diagnostic imaging [5]. In Nanomedicine, researchers are dedicating efforts to provide safe and efficient drug delivery systems with drug-loaded magnetic nanoparticles resulting in more specific and localized treatments. This is especially important for cancer therapy because it would benefit from magnetically-guided chemotherapy with highly toxic drugs reaching the tumor site without prejudice other tissues. 
Nanoparticles applications in medicine are extensive, as demonstrated by the high number of approaches under scientific investigation; some examples in addition to drug delivery, gene delivery, immobilization of peptides, proteins and other biomolecules; diagnostic tests; biological indicators; pathogenic biodetectors; hyperthermia and separation and purification of molecules [3-6]. Combination of drug delivery and magneticallyinduced hyperthermia, in which the application of an alternating magnetic field increases the local temperature until $42-45{ }^{\circ} \mathrm{C}$, has been revealed as an extremely efficient approach to treat glioblastoma and in general by others tumors in vivo [4]. Besides, it has been demonstrated that functionalization of magnetic nanoparticles with compounds of low solubility and/or a high degree of toxicity improves drug availability and decreases systemic toxicity [4]. Iron oxide magnetic nanoparticles are usually synthesized by chemical methods (co-precipitation, oxidation, etc.) [7]. Magnetite nanoparticles can also be produced a group of bacteria, called magnetotactic bacteria, in a genetically controlled process of biomineralization (Figure 1). The precise control over the intracellular synthesis of these nanostructures, named magnetosomes, provides them superior characteristics in comparison to artificially synthesized particles [4], such as a narrow size range, specific morphology, chemical purity and a permanent magnetic moment.
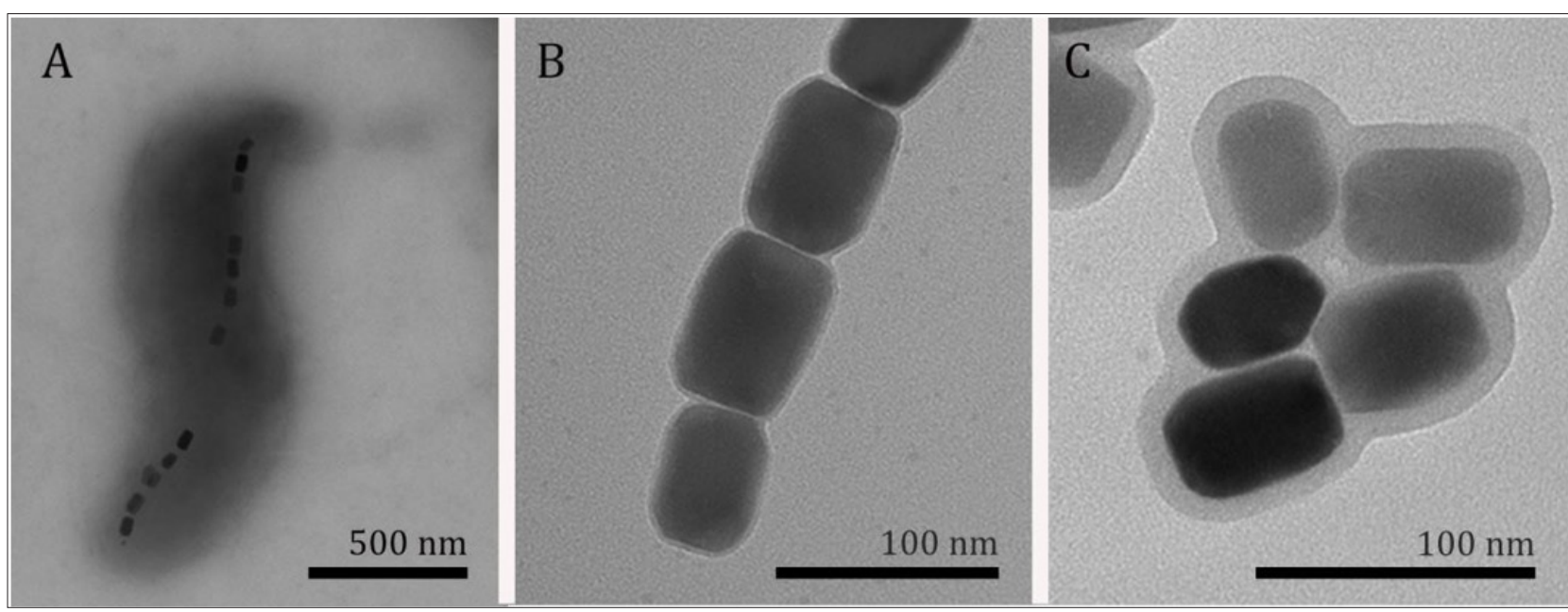

Figure 1: Transmission electron microscopy of magnetotactic bacteria.

Note:

A. purified magnetosomes

B. and drug-functionalized magnetosomes

C. Note that the increase of the thickness of the magnetosome membrane after the functionalization.

In addition, these structures are surrounded by a lipid bilayer containing proteins, which means that these nanoparticles are naturally biocompatible and easily functionalized [4]. An additional advantage is that the production of these nanoparticles during bacterial growth is an entirely green technology. That being said, it is clear that magnetosomes present significant advantages and potential of applicability in the development of new drug carriers and formulation for treatments. However, one of the drawbacks for some approaches might be that the biologically produced nanoparticles are permanently magnetic and their effects on a biological system have not been deeply evaluated. Regardless of biological or chemical synthesis, the most critical consideration for the application of magnetic nanoparticles in biomedicine is the toxic effect that these nanostructures could cause to humans. In the particular case of biologically synthesized nanoparticles, the process of isolation includes the lysis of the bacterial cell, which might release outer membrane lipopolysaccharide. Although the non-pyrogenic property of extracted magnetosomes have been confirmed [8], tests for endotoxin presence are always necessary to ensure quality and safe use of these nanoparticles. The major cytotoxicity test evaluated in in vitro experiments is the MTT assay that assesses the metabolism of cells after interaction of nanoparticles.
Other tests, for example, LDH assay to measure membrane lysis or Annexin/PI to see apoptosis and necrosis should also be considered before in vivo evaluation. Another critical point is that only a few human cell lineages (macrophages, fibroblast and tumors) were tested concerning cytotoxicity of these nanoparticles [4]. The human body is composed of several cell types that respond to external stimulus differently, so it is necessary to consider the toxic effects of magnetic nanoparticles in other cellular models. Therefore, it is crucial that before the approval for clinical use, or even in vivo assays, those nanoparticles should be evaluated by hemolysis, coagulation, and neurotoxicity tests. The introduction of iron oxide nanoparticles into human body raises a series of relevant questions. How the organism process those particles? How long they persist in the organism? Are they dissolved by the acid environmental inside of the cell and eliminated in the urine and/or feces? Alternatively, could the cell use them as an iron source? It is worth mentioning that a few researchers investigated the magnetic nanoparticles toxicity using in vitro and in vivo at least in the short term. However, the toxicity in long-term exposure should be carried out to reveal any side effect. An alternative for the preliminary in vivo test could be the use of Galleria mellonella as an experimental model, which is considered a cheap and easy in vivo assay [9]. This model has become very popular in recent years, especially for the 
study of the pathogenicity and virulence of microorganisms, as well as for the evaluation of antimicrobial compounds.

\section{Conclusion}

Extensive research focused on the biocompatibility and biological outcome of bacterial magnetic nanoparticles and derived formulations in both in vitro and in vivo models are still to be done. There is a considerable amount of information available about artificial nanoparticles in biological systems, which may be used to guide experimental protocols and predict results of the effects of bacterially-produced nanoparticles. Strategies for cultivating magnetotactic bacteria in large scale and the establishment of nanoparticles extraction and sterilization processes are also mandatory for the production of these next generation formulations in industrial settings. Finally, established protocols for quality assurance have to be developed to ensure safe use of these nanotechnological products (i.e. purity, sterility and pyrogenic testing). Once these conditions are met, clinical trials will be made tangible and will provide us with reliable information on the systemic and long-term effects of magnetic nanoparticles in patients. The Greek philosopher Thales of Miletus believed that, because magnets move iron, the matter contained life. Nowadays, magnetic nanoparticles inspire the scientific community to explore this incredible property over matter to save lives.

\section{Acknowledgment}

This research was financed in part by the by CAPES, FAPERJ and CNPq.

\section{ISSN: 2574-1241}

DOI: 10.26717/BJSTR.2018.11.002091

Fernanda Abreu. Biomed J Sci \& Tech Res

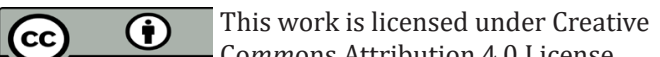

Submission Link: https://biomedres.us/submit-manuscript.php

\section{References}

1. Krukemeyer MG, KV Huebner F, Wagner W, Resch R (2015) History and possible uses of nanomedicine based on nanoparticles and nanotechnological progress. J Nanomed Nanotechnol 6(6): 336.

2. Reibold M, Paufler P, Levin AA, Kochmann W, Pätzke N (2006) Carbon nanotubes in an ancient damascus sabre. Nature 444 (7117): 286.

3. Berry CC (2012) Applications of inorganic nanoparticles for biotechnology. Frontiers of nanoscience Amsterdam Elsevier 4: 159180 .

4. Vargas G, Cypriano J, Correa T, Leão P, Bazylinski D, et al. (2018) Applications of magnetotactic bacteria, magnetosomes and magnetosome crystals in biotechnology and nanotechnology: Minireview. Molecules 23(10): 2438.

5. Rosen JE, Yoffe S, Meerasa A, Verma M, Gu FX (2011) Nanotechnology and diagnostic imaging: new advances in contrast agent technology. J Nanomed Nanotechnol 2(5): 1-12.

6. Salata OV (2004) Applications of nanoparticles in biology and medicine. Journal of nanobiotechnology 2: 3 .

7. Lu AH, Salabas EL, Schüth F (2004) Magnetic nanoparticles: Synthesis, protection, functionalization, and application. Angewandte Chemie International Edition 46: 1222-1244.

8. Sun J, Tang T, Duan, J, Xu PX, Wang Z, et al. (2010) Biocompatibility of bacterial magnetosomes: Acute toxicity, immunotoxicity and cytotoxicity. Nanotoxicology 4(3): 271-283.

9. Renwick J, Daly P, Reeves EP, Kavanagh K (2006) Susceptibility of larvae of Galleria mellonella to infection by Aspergillus fumigatus is dependent upon stage of conidial germination. Mycopathologia 161(6): 377-384.

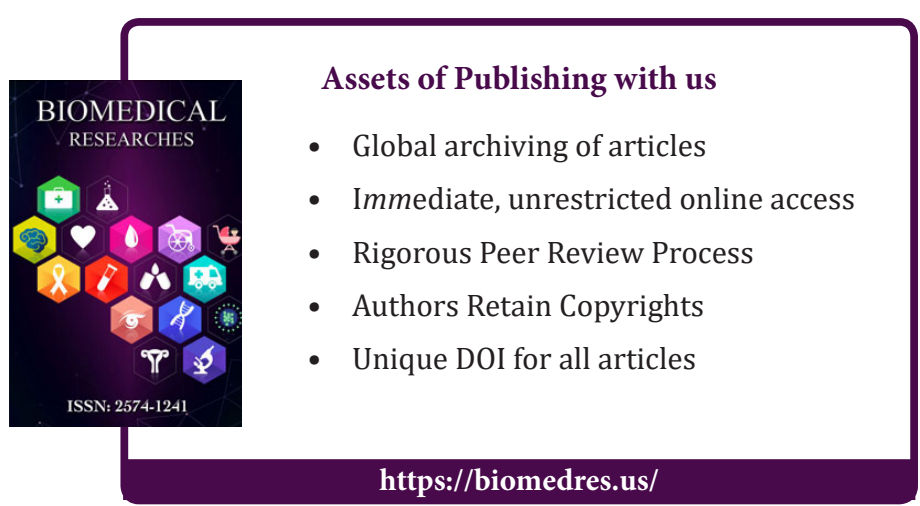

